\title{
Orbit equivalence and permutation groups defined by unordered relations
}

\author{
Francesca Dalla Volta $\cdot$ Johannes Siemons
}

Received: 8 July 2011 / Accepted: 2 September 2011 / Published online: 23 September 2011

(C) Springer Science+Business Media, LLC 2011

\begin{abstract}
For a set $\Omega$ an unordered relation on $\Omega$ is a family $R$ of subsets of $\Omega$. If $R$ is such a relation we let $\mathcal{G}(R)$ be the group of all permutations on $\Omega$ that preserve $R$, that is $g$ belongs to $\mathcal{G}(R)$ if and only if $x \in R$ implies $x^{g} \in R$. We are interested in permutation groups which can be represented as $G=\mathcal{G}(R)$ for a suitable unordered relation $R$ on $\Omega$. When this is the case, we say that $G$ is defined by the relation $R$, or that $G$ is a relation group. We prove that a primitive permutation group $\neq$ $\operatorname{Alt}(\Omega)$ and of degree $\geq 11$ is a relation group. The same is true for many classes of finite imprimitive groups, and we give general conditions on the size of blocks of imprimitivity, and the groups induced on such blocks, which guarantee that the group is defined by a relation.

This property is closely connected to the orbit closure of permutation groups. Since relation groups are orbit closed the results here imply that many classes of imprimitive permutation groups are orbit closed.
\end{abstract}

Keywords Group invariant relations · Regular sets · Orbit closure · Automorphism groups of set systems

\section{Introduction}

Let $\Omega$ be a set. Then the symmetric group $\operatorname{Sym}(\Omega)$ acts naturally on the collection $P(\Omega)$ of all subsets of $\Omega$. If $R$ is a subset of $P(\Omega)$ we may define the group

$$
\mathcal{G}(R):=\left\{g \in \operatorname{Sym}(\Omega): x^{g} \in R \text { for all } x \in R\right\}
$$

F. Dalla Volta $(\bowtie)$

Dipartimento di Matematica e Applicazioni, Università Milano Bicocca, 20125 Milano, Italy

e-mail: francesca.dallavolta@unimib.it

J. Siemons

School of Mathematics, University of East Anglia, Norwich, NR4 7TJ, UK

e-mail: j.siemons@uea.ac.uk 
of all permutations that leave $R$ invariant. In this paper we are interested in the permutation groups which arise in this way. We consider $R$ as an unordered relation, or just a relation on $\Omega$, and we say that a permutation group $G$ on $\Omega$ is a relation group if there is a relation $R$ on $\Omega$ so that $G=\mathcal{G}(R)$. The notion of relation group has appeared earlier in the literature, see for instance Betten's paper [4]. Our first result is Theorem 4.2 where we show that a finite primitive group $G$ on $\Omega$ is a relation group unless $G$ is $\operatorname{Alt}(\Omega)$ or one of ten exceptions of degree $\leq 10$. The remainder of the paper therefore deals with finite imprimitive groups. In Corollary 6.2 it is shown that most imprimitive groups are relation groups, in the following sense: If $H$ is an imprimitive group of degree $n$ with a block of imprimitivity $\Delta$ so that the group induced by $H$ on $\Delta$ does not contain $\operatorname{Alt}(\Delta)$ and so that $\Delta$ is sufficiently large in comparison to $n|\Delta|^{-1}$ then all subgroups of $H$ are relation groups. In Theorem 7.1 the same conclusion is obtained if some imprimitivity chain for $H$ does not include factors belonging to a certain class of groups defined there. This allows us also to deal efficiently with solvable groups.

This study of relation groups arose from a closely connected property of permutation groups. Two groups on $\Omega$ are called orbit equivalent if they have the same orbits on $P(\Omega)$. From this the orbit closure of $G$ is defined as the largest group $G^{*}$ on $\Omega$ that is orbit equivalent to $G$, and $G$ is orbit closed if $G=G^{*}$. Orbit equivalence has been studied for some time, and we give some references in the text. From the classification of finite simple groups there is a complete list of primitive pairs of orbit equivalent groups due to Seress [14] based on an observation by Cameron, Neumann and Saxl [5]. More recently Seress and Young [15] investigate the case when one of the groups is the wreath product of two primitive groups.

It is almost immediate that a relation group is orbit closed, see Proposition 2.5. The results mentioned above therefore show that large classes of permutation groups are orbit closed. In fact, we show that a primitive group is orbit closed if and only if it is a relation group. This, however, fails for infinitely many imprimitive groups, see Corollary 5.2.

Independently of this application relation groups are interesting in their own right: They include the full automorphism groups of graphs, designs, geometries and undirected set systems generally. Our main tool for dealing with relation groups are regular sets for primitive groups, and this requires the classification of finite simple groups. We also require the explicit knowledge of primitive groups without regular sets in [14]. In the last section we mention open problems for relation groups and orbit closure.

\section{Prerequisites}

Throughout $\Omega$ denotes a set of size $n$ and $P(\Omega)$ denotes the collection of all subsets of $\Omega$. For $0 \leq k \leq n$ the $k$-element subsets of $\Omega$ are denoted by $\Omega^{\{k\}}$. All sets and groups in this paper are finite.

\subsection{Action on subsets}

The symmetric group $\operatorname{Sym}(\Omega)$ acts naturally on $\mathrm{P}(\Omega)$ via $g: x \mapsto x^{g}:=\left\{\omega^{g}: \omega \in x\right\}$ for $g \in \operatorname{Sym}(\Omega)$ and $x \in \mathrm{P}(\Omega)$. Let $(G, \Omega)$ be a permutation group on $\Omega$ and let 
$x \in \mathrm{P}(\Omega)$. Then $x^{G}=\left\{x^{g}: g \in G\right\}$ is the $G$-orbit of $x$. We say that $G$ is set-transitive if $G$ acts transitively on $\Omega^{\{k\}}$ for all $k=0, \ldots, n$. We use standard ATLAS notation for groups, in particular $C_{m}, D_{m}$ and $F_{m}$ are the cyclic, dihedral and Frobenius groups of order $m$, respectively. We write also $S_{m}=\operatorname{Sym}(\Gamma)$ and $A_{m}=\operatorname{Alt}(\Gamma)$ when $|\Gamma|=m$.

Lemma 2.1 (Beaumont and Peterson [3], 1955) Let $H \subseteq \operatorname{Sym}(\Omega)$ be a set-transitive group of degree $n \geq 2$. Then $H \supseteq \operatorname{Alt}(\Omega)$ or

(i) $n=5$ and $H=F_{20}$,

(ii) $n=6$ and $H=L_{2}(5) .2 \simeq S_{5}$ or

(iii) $n=9$ and $H \in\left\{L_{2}(8) \subseteq L_{2}(8) .3\right\}$.

\subsection{Regular sets}

Let $(G, \Omega)$ be a permutation group on $\Omega$. We denote the setwise stabilizer of $x$ in $G$ by $G_{x}$. Then $x$ is a regular set for $G$ if $G_{x}=1$. A key tool for this paper is the characterization of primitive groups without a regular set. This is obtained from the classification of finite simple groups. We state this result in full as we need the details later on. The set of primitive groups of degree $5 \leq n \leq 32$ in the theorem without regular set will be denoted by $\mathcal{L}_{\mathrm{NR}}$.

Theorem 2.2 (Seress [14]) Let $H \subseteq \operatorname{Sym}(\Omega)$ be a primitive group of degree $n \geq 2$. Suppose that $H$ is not set-transitive. Then $H$ has a regular set on $\Omega$ if and only if $H$ is not one of the following:

(i) $n=5$ with $H=D_{10}$;

(ii) $n=6$ with $H=L_{2}(5)$;

(iii) $n=7$ with $H=F_{42}, H=L_{3}(2)$;

(iv) $n=8$ with $H \in\left\{2^{3}: 7.3 \subset 2^{3}: L_{3}(2)\right\}, H \in\left\{L_{2}(7) \subset L_{2}(7) .2\right\}$;

(v) $n=9$ with $H \in\left\{3^{2}:\left(2 . L_{2}(3)\right) \subset 3^{2}: 2 . L_{2}(3) .2\right\}, H \in\left\{3^{2}: D_{8} \subset 3^{2}: 8.2 \subset 3^{2}\right.$ :

2. $\left.L_{2}(3) .2\right\}$

(vi) $n=10$ with $H \in\left\{S_{5}, L_{2}(9) \subset L_{2}(9) .2 \subset L_{2}(9) .2 .2\right\}$,

$H \in\left\{L_{2}(9) \simeq P S U(2,9) \subset P G U(2,9)\right\}, H \in\left\{L_{2}(9) \subset M_{10}\right\}$;

(vii) $n=11$ with $H \in\left\{L_{2}(11) \subset M_{11}\right\}$;

(viii) $n=12$ with $H=L_{2}(11) .2, H \in\left\{M_{11} \subset M_{12}\right\}$;

(ix) $n=13$ with $H=L_{3}(3)$;

(x) $n=14$ with $H=L_{2}(13) .2$;

(xi) $n=15$ with $H=L_{4}(2) \simeq A_{8}$;

(xii) $n=16$ with $H=2^{4}:\left(A_{5} \times 3\right) .2, H \in\left\{2^{4}: A_{6} \subset 2^{4}: S_{6} \subset 2^{4}: L_{4}(2)\right\}$,

$H \in\left\{2^{4}: A_{7} \subset 2^{4}: L_{4}(2)\right\}$;

(xiii) $n=17$ with $H \in\left\{L_{2}(16) .2 \subseteq L_{2}(16) .4\right\}$;

(xiv) $n=21$ with $H=L_{3}(4) .3 .2$;

(xv) $n=22$ with $H \in\left\{M_{22} \subseteq M_{22} .2\right\}$;

(xvi) $n=23$ with $H=M_{23}$;

(xvii) $n=24$ with $H=M_{24}$;

(xviii) $n=32$ with $H=2^{5}: L_{5}(2)$. 
In each case the containments of the primitive exceptions are as indicated. In particular, a primitive, not set-transitive group of degree $n \notin\{5,6,7,8,9,10,11,12,13$, $14,15,16,17,21,22,23,24,32\}$ has a regular set.

Comments 1. The theorem requires the classification of finite simple groups in the following way: If $(G, \Omega)$ is a permutation group without regular sets then each of the $2^{|\Omega|}$ subsets of $\Omega$ is stabilized by a non-identity element. Since a permutation composed of $c$ cycles stabilizes exactly $2^{c}$ subsets, a bound for the minimum degree in $G$ (the least number of elements by a non-identity element in $G$ ) implies that $G$ is 'large'. On the other hand, from the O'Nan-Scott Theorem and the classification of finite simple groups an upper bound for the order of a primitive group not containing the alternating group is obtained, and this yields an upper bound for the order of $G$. This argument due to Cameron, Neumann and Saxl [5] shows that all but finitely many primitive groups not containing the alternating group of the same degree have a regular set. In Sect. 7 we use the same idea to prove that all but finitely many such groups have regular sets of at least two different cardinalities. The list $\mathcal{L}_{\mathrm{NR}}$ of exceptions is obtained in Seress' theorem by further careful analysis of the bounds and by direct computations.

2. There are direct methods to establish the existence of a regular set for many classes of permutation groups, and these methods do not require primitivity or the classification. If the group is given as the automorphism group of a geometrical or combinatorial object, say a graph, an affine or projective space, or a design more generally, it is often possible to construct a regular set from the combinatorics of the structure. In the literature on graphs and set systems the more general notion of a distinguishing partition appears, introduced by Albertson and Collins [1]. These are partitions into an arbitrary number of classes so that only the identity automorphism stabilizes all classes, see the paper [12] by C. Laflamme, Nguyen Van Thé and N. Sauer. Regular sets therefore correspond to distinguishing partitions into two classes.

3. We mention some papers where regular sets are obtained by such direct methods. Gluck [8] determines all solvable primitive groups without a regular set. In $[6,7,9,10]$ it is shown that regular sets exist for the affine, projective, unitary or orthogonal groups in their natural action, apart from a small number of explicitly listed exceptions. Often the size of a smallest regular set is determined as well. The problem of determining the primitive groups in which for given $k$ all $k$-element subsets are regular is considered in Bates, Bundy, Hart and Rowley [2].

\subsection{Relations and relation groups}

We consider a subset $R$ of $P(\Omega)$ as an unordered relation on $\Omega$, or just a relation. (We shall not consider the usual ordered relations.) If $R$ is a relation then

$$
\mathcal{G}(R):=\left\{g \in \operatorname{Sym}(\Omega): x^{g} \in R \text { for all } x \in R\right\}
$$

is the invariance group of $R$, or the group defined by $R$. Evidently a permutation group $G$ on $\Omega$ is contained in $\mathcal{G}(R)$ if and only if $R$ is a union of $G$-orbits on $P(\Omega)$. We call $G$ a relation group on $\Omega$ if there is some $R$ on $\Omega$ for which $G=\mathcal{G}(R)$. It 
is also possible to view $R$ as a hypergraph with vertex set $\Omega$, and $\mathcal{G}(R)$ as its full automorphism group. In this paper we prefer, however, the language of relations as it is natural and more flexible.

Key examples of relation groups arise from combinatorial structures and geometry. For instance, if $\Omega$ is the set of points of a projective or affine space $\mathcal{S}$ and if $C \subset \Omega^{\{3\}}$ is the collinearity relation of $\mathcal{S}$ (i.e. the collinear triples) then $\operatorname{Aut}(\mathcal{S})=\mathcal{G}(C)$ is a relation group by definition. In fact, we note that most classical groups arise in this fashion. With this in mind Betten [4] calls a relation group also a geometric group. The problem of representing a permutation group by an unordered relation on the same set was also considered in [10].

If $R$ is a relation then its arity is the set $\operatorname{ar}(R)=\{|x|: x \in R\}$. The arity of a relation is important for our purpose. The following obvious fact will be used without further mention.

Lemma 2.3 If $R^{\prime}$ and $R^{\prime \prime}$ are relations with $\operatorname{ar}\left(R^{\prime}\right) \cap \operatorname{ar}\left(R^{\prime \prime}\right)=\emptyset$ then $\mathcal{G}\left(R^{\prime} \cup R^{\prime \prime}\right)=$ $\mathcal{G}\left(R^{\prime}\right) \cap \mathcal{G}\left(R^{\prime \prime}\right)$.

We call $R$ trivial if $R=\bigcup_{k \in I} \Omega^{\{k\}}$ for some index set $I \subseteq\{0,1, \ldots, n\}$. Evidently $G$ is set-transitive if and only if the only relations $R$ with $G \subseteq \mathcal{G}(R)$ are the trivial relations.

\subsection{Orbit equivalence}

Let $0 \leq k \leq n$. Two permutation groups $G$ and $H$ on $\Omega$ are $k$-orbit equivalent if they have the same orbits on $\Omega^{\{k\}}$. We denote this by $G \approx^{k} H$. The largest permutation group on $\Omega$ that is $k$-orbit equivalent to $G$ is the $k$-closure of $G$, denoted $G^{\{k\}}$, and $G$ is $k$-orbit closed, or just $k$-closed if $G=G^{\{k\}}$. Evidently $G^{\{k\}}=G^{\{n-k\}}$. The following theorem states the relationship between the closure groups:

Theorem 2.4 (Siemons [16]) Let $G$ and $H$ be permutation groups on $\Omega$ and suppose that $0 \leq k \leq \ell$ satisfy $k+\ell \leq|\Omega|$. Then $G \approx^{\ell} H$ implies $G \approx^{k} H$. In particular, $G^{\{\ell\}} \subseteq G^{\{k\}}$ and so a $k$-closed group is $\ell$-closed.

We say that $G$ and $H$ are orbit equivalent, denoted $G \approx H$, if $G \approx^{k} H$ for all $0 \leq k \leq n$. The orbit closure of $G$ is the largest permutation group $G^{*}$ on $\Omega$ that is orbit equivalent to $G$. We say that $G$ is orbit closed if $G=G^{*}$. From the theorem it follows that $G^{*}=G^{\left\{n^{*}\right\}}$ where $n^{*}=\left\lfloor\frac{n}{2}\right\rfloor$. There are other closure operations for permutations groups in the literature. These are derived from the group action on the Cartesian product $\Omega^{k}$, rather than $\Omega^{\{k\}}$, and were first explored in Wielandt's Ohio lecture notes [17]. While there are interesting common features, we emphasize that in this paper we are not concerned with this notion of closure.

The connection between orbit closure and relation groups, of key importance in this paper, is immediate. We have

Proposition 2.5 Let $G$ be a permutation group on $\Omega$ and let $R$ be a relation with $G \subseteq$ $\mathcal{G}(R)$. Suppose that $1 \leq k \leq \frac{n+1}{2}$ is an integer so that no set $x$ in $R$ has cardinality $k<|x|<n-k$. Then $G^{\{k\}} \subseteq \mathcal{G}(R)$. In particular, a relation group is orbit closed. 
Proof By Theorem 2.4 the groups $G$ and $G^{\{k\}}$ have the same orbits on $t$-element subsets for all $t \leq k$ and $n-k \leq t$. Since $G \subseteq \mathcal{G}(R)$ if and only if $R$ is a union of $G$-orbits on $P(\Omega)$ we have $G^{\{k\}} \subseteq \mathcal{G}(R)$ provided that no set $x$ in $R$ has cardinality $k<|x|<n-k$.

Comments 1 . For small $k$ there are in some sense 'only few' partitions of $\Omega^{\{k\}}$ that arise as the orbits of a group on $\Omega$, and therefore 'only few' groups that are $k$-orbit closed. To be $k$-closed for small $k$ is therefore a strong property of permutation groups. For instance, $G$ is 1-closed if and only if $G=\operatorname{Sym}\left(\Omega_{1}\right) \times \cdots \times \operatorname{Sym}\left(\Omega_{t}\right)$ with $\Omega=\Omega_{1} \cup \cdots \cup \Omega_{t}$, and $G$ is 2-closed if and only if there is a (vertex and edge) coloured undirected graph on $\Omega$ so that $G$ is its full automorphism group. Much the same can be said about relation groups $\mathcal{G}(R)$ where the arity of $R$ is bounded by $k$ as in the proposition. So also in this case, to be the group of such a bounded relation is a strong property of permutation groups.

2. There are transitive orbit closed groups that are not relation groups. The smallest is the Klein 4-group $V$ on $\Omega=\{1,2,3,4\}$. It is transitive on sets of size $\neq 2$ and has 3 orbits on 2 -sets. It is easy to check that $V$ is orbit closed. Each of the three orbits on 2-sets gives a relation, and together with their complements these are the only non-trivial relations preserved by $V$. However, for any choice of an orbit (or its complement) there is a transposition not in $V$ that stabilizes the orbit. Hence $V \subset$ $\mathcal{G}(R)$ for any relation preserved by $V$. In Sect. 5 we shall show that there are infinitely many imprimitive groups of this kind.

3. In Sect. 4 (Corollary 4.3) we shall prove that a primitive group is a relation group if and only if it is orbit closed. It is an open problem to formulate general conditions for an orbit closed imprimitive group to be a relation group, see also Corollary 5.3.

The primitive orbit closed groups have been determined from Lemma 2.1 and Theorem 2.2:

Theorem 2.6 (Seress [14]) Let $G \subset H$ be primitive and orbit equivalent permutation groups on $\Omega$. Assume that $\{G, H\} \neq\{\operatorname{Alt}(\Omega), \operatorname{Sym}(\Omega)\}$. Then

(i) $|\Omega|=5$ when $\{G, H\}=\left\{C_{5}, D_{10}\right\}$ or $\{G, H\} \subseteq\left\{F_{20}, A_{5}, S_{5}\right\}$,

(ii) $|\Omega|=6$ when $\{G, H\} \subseteq\left\{L_{2}(5) .2 \simeq S_{5}, A_{6}, S_{6}\right\}$,

(iii) $|\Omega|=8$ when $\{G, H\} \subseteq\left\{2^{3}: 7 \subset 2^{3}: 7.3 \subset 2^{3}: L_{3}(2)\right\}$,

(iv) $|\Omega|=9$ when $\{G, H\}=\left\{3^{2}: 8 \subset 3^{2}: 8.2\right\},\{G, H\}=\left\{3^{2}: 2 . L_{2}(3) \subset 3^{2}\right.$ :

2. $\left.L_{2}(3) .2\right\}$ or $\{G, H\} \subseteq\left\{L_{2}(8), L_{2}(8) .3, A_{9}, S_{9}\right\}$,

(v) $|\Omega|=10$ when $\{G, H\}=\left\{L_{2}(9) .2 \subset L_{2}(9) .2 .2\right\}$.

\section{Subgroups of relation groups}

Under suitable conditions all subgroups of a relation group are again relation groups. This important and easy to prove property is the subject of this section. Recall that if $R$ is a relation on $\Omega$ then $\operatorname{ar}(R)=\{|x|: x \in R\}$ is the arity of $R$.

Basic Lemma 3.1 Let $H$ be a permutation group on $\Omega$ and suppose that $H$ has a regular set $w$. Suppose that one of the following holds: 
(i) There is a relation $R$ on $\Omega$ with $H=\mathcal{G}(R)$ so that $|w|$ does not belong to $\operatorname{ar}(R)$, or

(ii) $H$ is not set-transitive, and is a maximal subgroup of $\operatorname{Sym}(\Omega)$ with respect to this property.

Let $G$ be a subgroup of $H$. Then $G$ is a relation group on $\Omega$ and in particular, $G$ is orbit closed.

In this paper we are interested in showing that certain classes of permutation groups are relation groups, and hence are orbit closed. This is an important consequence of being a relation group. However, it makes no sense to repeat this on each occasion. In addition, with Proposition 2.5 in mind, even stronger closure properties hold for the group as soon as additional information about the arity of the relation in question in the Basic Lemma is available.

Proof (i) For $G \subseteq H$ let $R^{G}=w^{G}$ be the $G$-orbit of $w$ and let $r$ be the size of $w$. Then $G \subseteq \mathcal{G}\left(R \cup R^{G}\right)$ and since $r \notin \operatorname{ar}(R)$ we have $\mathcal{G}\left(R \cup R^{G}\right)=\mathcal{G}(R) \cap \mathcal{G}\left(R^{G}\right)=$ $H \cap \mathcal{G}\left(R^{G}\right)$ by Lemma 2.3. Let $h$ be an element of $H \cap \mathcal{G}\left(R^{G}\right)$. Then there is some $g \in G$ with $w^{h}=w^{g}$. Hence $h g^{-1} \in H$ stabilizes $w$ so that $h g^{-1}=1$ and so $h=g$ belongs to $G$. Therefore $G=\mathcal{G}\left(R \cup R^{G}\right)$.

(ii) As $H$ is not set-transitive let $x$ be a set with $x^{H} \neq \Omega^{\{k\}}$ where $k:=|x|$. Then $H \subseteq \mathcal{G}\left(x^{H}\right)$ and the latter is not set-transitive. By the maximality condition, $H=$ $\mathcal{G}\left(x^{H}\right)$. If $x$ can be chosen so that $k \neq r:=|w|$ the result follows from (i). It remains to consider the case when $H$ is transitive on $\ell$-sets for all $\ell \neq r$ but intransitive on $r$-sets. For any permutation group on $\Omega$, by the Livingstone-Wagner Theorem, or by Theorem 2.4, transitivity on $t$-sets implies transitivity on $s$-sets provided $s+t \leq|\Omega|$. So in our case $\Omega$ must have size $2 r$. As $H$ is transitive on $(r-1)$-sets we have $|H|=\left(\begin{array}{c}2 r \\ r-1\end{array}\right) \cdot\left|H_{v}\right|$ for any set $v$ of size $r-1$. If $\left|H_{v}\right|=1$ we may replace $w$ by $v$, and the result follows again from (i). Hence we may assume that $2\left(\begin{array}{c}2 r \\ r-1\end{array}\right) \leq|H|$. But we also have $|H|=\left|w^{H}\right|<\left(\begin{array}{c}2 r \\ r\end{array}\right)$ since $H$ is intransitive on $r$-element subsets. This implies $r=0$, a contradiction. Finally, if $G$ is a relation groups then $G$ is orbit closed by Proposition 2.5 .

Comments 1. This lemma is useful in many situations, we give some examples. Let $H$ be the group of all collineations of an affine or projective space of dimension $d$ over the field $F$, viewed as a permutation group on the set $\Omega$ of the points of the space. Let $C \subset \Omega^{\{3\}}$ be the collinearity relation. Then $H=\mathcal{G}(C)$, by definition. As mentioned in the comments following Theorem 2.2, a regular set $w$ for $H$ can be constructed by elementary means, and typically $w$ has cardinality $r=2 d$ or $r=2 d \pm 1$. There are some exceptions for small $d$ and $|F|$, the details are given in Theorems 3.1 and 4.1 of [9]. From this information we conclude that any subgroup $G$ of $H$ can be expressed as $G=\mathcal{G}\left(C \cup w^{G}\right)$. This relation is quite simple: only two set sizes are needed. It is evident that $C$ can be replaced by other homogeneous relations $C^{*}$ which encapsulate collinearity. We note that it is even possible to find a relation $C^{*} \subset \Omega^{\{r\}}$ so that $\mathcal{G}\left(C^{*} \cup w^{G}\right)=\mathcal{G}\left(C^{*}\right) \cap \mathcal{G}\left(w^{G}\right)$, see [10]. For this choice $G=\mathcal{G}\left(C^{*} \cup w^{G}\right)$ is the group of a homogeneous relation. 
This example shows that all subgroups of $A \Gamma L(d, F)$, and all subgroups of $P \Gamma L(d, F)$, are $r$-closed in their action on the points of the corresponding affine or projective space, apart from a few exceptions for small dimension $d$ and small field $F$. This follows from Proposition 2.5, and by Theorem 2.4 we may take $r=2 d+1$. The small exceptions are covered in Theorem 4.2.

2 . It is essential to control the arity of the relations that define a given group. Especially important is the case $|\operatorname{ar}(R)|=1$, when $R$ is homogeneous. In a slightly different setting groups defined by a homogeneous relation appear as 2-representable groups in Kisielewicz's paper [11]. In the second part of the Basic Lemma $H$ is the group of a homogeneous relation, for if $v^{H}$ is any non-trivial $H$-orbit then $H=$ $\mathcal{G}\left(v^{H}\right)$. If $G \subset H$ then the proof has shown that $G=\mathcal{G}(R)$ for a relation $R$ with $|\operatorname{ar}(R)|=2$.

\section{Primitive groups}

In this section we consider primitive groups. As a first application of the Basic Lemma we prove that they all are relation groups apart from a few exceptions.

Theorem 4.1 Let $H$ be a primitive permutation group on $\Omega$ not containing $\operatorname{Alt}(\Omega)$. Suppose that $|\Omega|>9$ and that $H$ does not belong to the list $\mathcal{L}_{\mathrm{NR}}$ of Theorem 2.2. Then every subgroup of $H$ is a relation group.

Remark In the remaining case, when $H$ does belong to $\mathcal{L}_{\mathrm{NR}}$, it is straightforward to determine all relation groups contained in $H$ by direct computation. However, we omit these details here.

Proof As $|\Omega|>9$ it follows from Lemma 2.1 that $H$ is not set-transitive and as $H$ is not in $\mathcal{L}_{\mathrm{NR}}$ it follows that $H$ has a regular set. Without loss of generality we may suppose that $H$ is maximal with the property of not being set-transitive. Now apply the second part of the Basic Lemma.

Our next aim is to determine the primitive permutation groups on $\Omega$ that are not the group of a relation on $\Omega$. We describe these exceptions. In our lists $(n, H)$ means that $H$ is a group of degree $n=|\Omega|$.

Set-transitive groups: If $H \neq \operatorname{Sym}(\Omega)$ is a set-transitive group then $H$ is not a relation group. Apart from $H=\operatorname{Alt}(\Omega)$ by Lemma 2.1 the only groups of this type are

$$
\mathcal{L}_{\mathrm{ST}}=\left\{\left(5, F_{20}\right),\left(6, L_{2}(5) .2\right),\left(9, L_{2}(8)\right),\left(9, L_{2}(8) .3\right)\right\} .
$$

Note that none of these groups have a regular set.

Other orbit equivalent primitive groups: Suppose that $H_{1} \subset H_{2} \neq H_{1}$ are orbit equivalent, and not set-transitive. Then $H_{1}$ is not a relation group, by Proposition 2.5. From Theorem 2.6 we obtain the following possibilities for $H_{1}$ :

$$
\mathcal{L}_{\mathrm{OE}}=\left\{\left(5, C_{5}\right),\left(8,2^{3}: 7\right),\left(8,2^{3}: 7.3\right),\left(9,3^{2}: 8\right),\left(9,3^{2}: 2 . L_{2}(3)\right),\left(10, L_{2}(9) .2\right)\right\} .
$$


The groups $\left(8,2^{3}: 7.3\right),\left(9,3^{2}: 2 . L_{2}(3)\right)$ and $\left(10, L_{2}(9) .2\right)$ have no regular sets while the remaining three groups have regular sets.

Let $\mathcal{L}=\mathcal{L}_{\mathrm{ST}} \cup \mathcal{L}_{\mathrm{OE}}$. None of these groups is a relation group, and together with the alternating groups this determines all primitive groups which are not relation groups:

Theorem 4.2 Let $H$ be a primitive permutation group on $\Omega$ not containing $\operatorname{Alt}(\Omega)$. Then $H$ is a relation group if and only if $H$ does not belong to $\mathcal{L}$.

Corollary 4.3 A primitive permutation group $H$ on $\Omega$ is orbit closed if and only if it is a relation group. If $H$ is not a relation group then $H=\operatorname{Alt}(\Omega)$ or $|\Omega| \leq 10$. In all these cases the index of $H$ in its orbit closure is divisible by 2.

Remark Note that primitivity is required. As mentioned before, the Klein 4-group is orbit closed on $\{1,2,3,4\}$ but not a relation group. We shall discuss other examples in the next sections.

Proof of Theorem 4.2 We have already shown that if $H$ belongs to $\mathcal{L}$ then $H$ is not orbit closed and hence not a relation group. Conversely suppose that $H$ is primitive on $\Omega$, does not contain $\operatorname{Alt}(\Omega)$ and does not appear in $\mathcal{L}$. Then $H$ is not set-transitive. Let $X$ be a group containing $H$ that is maximal subject to being not set-transitive. By maximality $X$ is a relation group. If $X$ has a regular set then the result follows from the second part of the Basic Lemma.

It remains to inspect the cases when $X$ has no regular set. As $X$ is primitive it is one of the maximal groups in the list $\mathcal{L}_{\mathrm{NR}}$ of exceptions in Theorem 2.2. (We have $H \subset X \neq H$ but $H$ may or may not have a regular set.) Using the GAP libraries of primitive groups one can now inspect the primitive subgroups of $X$. This yields a list of about 58 subgroups $H$. All can be shown to be relation groups or members of $\mathcal{L}$. In some cases there is a relation group $Y \supset H$ which does have a regular set, and in this case the Basic Lemma suffices to show that also $H$ is a relation group. In the remaining cases we have computed sufficiently many $H$-orbits on $P(\Omega)$ so that a relation $R$ can be stated explicitly for which $H=\mathcal{G}(R)$.

In the following we give the complete details of the computation for the groups of degree $n=16$, the other cases where studied in similar way. ${ }^{1}$ Here we need to consider only possibility $X=A G L(4,2)=2^{4}: L_{4}(2)$, the group of the planarity relation in $\mathrm{GF}_{2}^{4}$. It has 19 primitive subgroups, four of these have no regular set. We will now outline the proof for the fact that these are all groups of relations. (In brackets we state the GAP name of the group.)

There are three maximal subgroups of $X$, these are $H_{17}=2^{4}: S_{6}\left(=G_{16}\right), H_{18}=$ $2^{4}: A_{7}\left(=G_{20}\right)$ and $H_{19}=A \Gamma L(2,4)\left(=G_{12}\right)$. In each case we name an orbit of the group on subsets that is not an orbit of $X$ : For $H_{17}=\mathcal{G}\left(R_{17}\right)$ select an orbit $R_{17} \subset \Omega^{\{3\}}$ of length 240 , for $H_{18}=\mathcal{G}\left(R_{18}\right)$ select an orbit $R_{18} \subset \Omega^{\{5\}}$ of length 672 and for $H_{19}=\mathcal{G}\left(R_{19}\right)$ select an orbit $R_{19} \subset \Omega^{\{4\}}$ of length 20 .

\footnotetext{
${ }^{1}$ A full version of the paper containing the details for the primitive groups of degree $\leq 32$ is available on arXiv.org.1010.3536.
} 
We turn to groups that are maximal in one of the above. These are $H_{11}=$ $A \Gamma L(1,16)\left(=G_{9}\right), H_{12}=\left(S_{4} \times S_{4}\right): 2\left(=G_{10}\right), H_{13}=2^{4}: S_{5}\left(=G_{18}\right), H_{14}=2^{4}$ : $A_{6}\left(=G_{17}\right), H_{15}=A S L(2,4): 2\left(=G_{13}\right), H_{16}=A G L(2,4)\left(=G_{14}\right)$. The containments are $H_{12}, H_{13}, H_{14}, H_{15} \subset H_{17}$, further $H_{14}, H_{15} \subset H_{18}$ and $H_{11}, H_{15}, H_{16} \subset$ $H_{19}$. In each case we name an orbit of the group that is not an orbit of any group properly containing it.

For $H_{11}=\mathcal{G}\left(R_{11} \cup R_{19}\right) \subset H_{19}$ select an $H_{11}$-orbit $R_{12} \subset \Omega^{\{5\}}$ of length 480 . For $H_{12}=\mathcal{G}\left(R_{12} \cup R_{17}\right) \subset H_{17}$ select an $H_{12}$-orbit $R_{12} \subset \Omega^{\{4\}}$ of length 8 . For $H_{13}=\mathcal{G}\left(R_{13} \cup R_{17}\right) \subset H_{17}$ select an $H_{13}$-orbit $R_{13} \subset \Omega^{\{4\}}$ of length 40 . We have $H_{14}=H_{17} \cap H_{18}=\mathcal{G}\left(R_{17} \cup R_{18}\right)$ and $H_{15}=H_{18} \cap H_{19}=\mathcal{G}\left(R_{18} \cup R_{19}\right)$. For $H_{16}=$ $\mathcal{G}\left(R_{16} \cup R_{19}\right) \subset H_{19}$ select an $H_{16}$-orbit $R_{16} \subset \Omega^{\{6\}}$ of length 1440 .

The remaining groups are contained in $H_{12}, H_{13}, H_{15}$ or $H_{16}$. Each of these has a regular set of size 6,7 or 8 . These set sizes are distinct from the set sizes of the relations defining $H_{12}, H_{13}, H_{15}$ and $H_{16}$. Hence the result follows from the Basic Lemma.

Proof of Corollary 4.3 If $H$ is a relation group then $H$ is orbit closed by Proposition 2.5. Conversely, by the comment above, if $H$ is orbit closed then it is not in $\mathcal{L}$ and so by Theorem 4.2 we see that $H$ is not a relation group. If $H$ is not orbit closed then by the theorem, either $H=\operatorname{Alt}(\Omega)$ or $H$ belongs to $\mathcal{L}$. In the latter case it is easy to check that the index of $G$ in its orbit closure is divisible by 2 , for this see again Theorem 2.6.

\section{Wreath products}

Let $K$ be a permutation group on the set $\Delta$ and let $L$ be a transitive permutation group on the set $\Sigma$. We are interested in the imprimitive action of $K$ \& $L$ on $\Omega:=\Delta \times \Sigma$. (This will be the only action considered here for wreath products.) First we show that the full wreath product $(K, L, \Omega)$ is a relation group if the same is true for $(K, \Delta)$ and $(L, \Sigma)$ when $(K, \Delta)$ is primitive. Imprimitive groups more generally will be considered in the next section. If $R$ is a relation then the maximum set size in $R$ is denoted by $m(R):=\max \{|x|: x \in R\}$.

Theorem 5.1 Let $\Delta$ and $\Sigma$ be sets and put $\Omega:=\Delta \times \Sigma$. Assume that $(K, \Delta)$ and $(L, \Sigma)$ are permutation groups where $K$ acts primitively.

(i) If $(K, \Delta)$ and $(L, \Sigma)$ are relation groups then $(K \geq L, \Omega)$ is a relation group.

(ii) If $(K \imath L, \Omega)$ is a relation group then $(K, \Delta)$ is a relation group.

In the first case, if $R^{\Sigma} \neq \emptyset$ is a relation for $L$ then there exists a relation $R^{\Omega}$ for $K$ ? $L$ with $m\left(R^{\Omega}\right)=|\Delta| m\left(R^{\Sigma}\right)$.

Corollary 5.2 Let $(K, \Delta)$ be a primitive permutation group and let $(L, \Sigma)$ be a relation group. Then $K$ ? $L$ is a relation group on $\Delta \times \Sigma$ if and only if $(K, \Delta)$ is not $\operatorname{Alt}(\Delta)$ and not one of the groups in the list $\mathcal{L}$ of Sect. 4. 
Comment It is essential here that we consider the full wreath product. As an illustration take $(K, \Delta)=\left(C_{2},\{1,2\}\right)=(L, \Sigma)$ when $C_{2}$ ? $C_{2}=D_{8}$ is a relation group on $\{1,2,3,4\}$. The Klein group $V \subset C_{2} 2 C_{2}$ is orbit closed, as we have seen earlier, but it is not a relation group. Note that $D_{8}$ has no regular set. This is not an isolated example:

Corollary 5.3 There are infinitely many wreath products $(K, L, \Omega)$ with both $(K, \Delta)$ and $(L, \Sigma)$ primitive such that $(K, L, \Omega)$ is orbit closed but not a relation group.

Proof of Theorem 5.1 For (i) let $\Sigma=\{1 . . s\}$ and denote $\Delta_{i}:=\Delta \times\{i\}$ where we identify $\Delta=\Delta_{1}$. Suppose that there is a relation $R^{\Delta}$ on $\Delta$ so that $K=\mathcal{G}\left(R^{\Delta}\right)$. Since $K$ is transitive we may assume that all sets $y$ in $R^{\Delta}$ have size $2 \leq|y| \leq d-2$. Correspondingly let $R^{\Delta_{i}} \subseteq P\left(\Delta_{i}\right)$ be a copy of $R^{\Delta}$ on $\Delta_{i}$. Let

$$
R^{\prime}=\left\{\Omega \backslash y: y \text { belongs to some } R^{\Delta_{i}}\right\} .
$$

Let $H=K 2 \operatorname{Sym}(\Sigma)$. We claim that $H=\mathcal{G}\left(R^{\prime}\right)$. Clearly, $H \subseteq \mathcal{G}\left(R^{\prime}\right)$. On $\Omega$ we define an adjacency relation $\alpha \sim \beta$ if and only if there is some $x \in R^{\prime}$ with $|\{\alpha, \beta\} \cap x|=0$. (In other words, $\alpha \sim \beta$ if and only if $\{\alpha, \beta\} \subseteq y$ for some $y \in R^{\Delta_{i}}$.) Evidently there is no edge from $\Delta_{i}$ to $\Delta_{j}$ if $i \neq j$, and as $\mathcal{G}\left(R^{\Delta_{i}}\right)$ is primitive $\Delta_{i}$ is a connected component of the graph, for $i=1, \ldots, s$. Therefore each element $g$ in $\mathcal{G}\left(R^{\prime}\right)$ permutes the $\Delta_{i}$ as sets, and if $g$ stabilizes $\Delta_{j}$ then it preserves $R^{\Delta_{j}}$. Hence $g \in H$ and therefore $\mathcal{G}\left(R^{\prime}\right)=H$. Now

$$
R=R^{\Delta_{1}} \cup \cdots \cup R^{\Delta_{s}}
$$

is the collection of all complements of sets in $R^{\prime}$ and so also $H=\mathcal{G}(R)$. All sets in $R$ have size $\leq d-2$.

Next let $R^{\Sigma}$ be a relation of $\Sigma$ with $L=\mathcal{G}\left(R^{\Sigma}\right)$ and suppose that the maximum set size in $R^{\Sigma}$ is $m_{\Sigma}$. Define

$$
R^{\prime \prime}=\left\{\Delta_{i_{1}} \cup \cdots \cup \Delta_{i_{t}}:\left\{i_{1} \cdots i_{t}\right\} \in R^{\Sigma}\right\} .
$$

The sets in $R^{\prime \prime}$ have size $\ell d$ where $1 \leq \ell \leq m_{\Sigma}$ and they are larger than any set in $R$. It follows that

$$
\mathcal{G}\left(R \cup R^{\prime \prime}\right)=\mathcal{G}(R) \cap \mathcal{G}\left(R^{\prime \prime}\right) .
$$

In particular, $K \imath L \subseteq \mathcal{G}\left(R \cup R^{\prime \prime}\right)$. Conversely, if $h \in \mathcal{G}\left(R \cup R^{\prime \prime}\right)$ then $h \in \mathcal{G}(R)=$ $H$, and so $h$ permutes the sets $\Delta_{i}$. Therefore $h$ induces a permutation $h_{\Sigma}$ of $\Sigma$ preserving $R^{\Sigma}$. Hence $h_{\Sigma} \in L=\mathcal{G}\left(R^{\Sigma}\right)$ and so $h$ belongs to $K$ ? $L$. Therefore $K$ ₹ $L=\mathcal{G}\left(R \cup R^{\prime \prime}\right)$ is a relation group on $\Omega$.

Conversely, for (ii) suppose that there is some relation $R$ on $\Omega$ so that $K$ ? $L=$ $\mathcal{G}(R)$. For each $i=1, \ldots, s$ consider the relation $R_{i}:=\left\{y \cap \Delta_{i}: y \in R\right\}$ and the subgroup of $K$ \& $L$ given by $K_{i}=\{(1, \ldots, 1, k, 1, \ldots, 1): k \in K\}$ with $k$ in position $i$. Then $K_{i}$ fixes $\Delta_{i}$ and preserves $R_{i}$ so that $K_{i} \subseteq \mathcal{G}\left(R_{i}\right)$. As $L$ is transitive the permutation groups $\left(K_{i}, \Delta_{i}\right)$ are permutationally isomorphic to each other for $i=$ $1, \ldots, s$. In particular, $K=K_{1} \subseteq \mathcal{G}\left(R_{1}\right)$ as a group on $\Delta=\Delta_{1}$. 
Assume that $K$ is not a relation group. From Corollary 4.3 it follows that $K$ is not orbit closed. Let therefore $K^{*} \neq K$ be its orbit closure, so that $K \subset K^{*} \subseteq G\left(R_{1}\right) \neq$ $K$ by Proposition 2.5. We show that this implies $K^{*} 2 L \subseteq \mathcal{G}(R)$, contradicting that $K \imath L=\mathcal{G}(R)$.

A subset $x$ of $\Omega$ is of the form $x=\bigcup_{i=1, \ldots, s}\left(x_{i}, i\right)$ with $x_{i} \subseteq \Delta$ and for $h=$ $\left(k_{1}, \ldots, k_{s} ; b\right) \in K$ ? $L$ we have $x h=\left(x_{1} k_{1}, 1 b\right) \cup \cdots \cup\left(x_{s} k_{s}, s b\right)$. To show that $K^{*}$ ? $L \subseteq \mathcal{G}(R)$ let $h^{*}=\left(k_{1}^{*}, \ldots, k_{s}^{*} ; b\right) \in K^{*}, L$ and let $x$ be a set in $R$. Then by the orbit equivalence of $K$ and $K^{*}$ there are elements $k_{1}, \ldots, k_{s}$ in $K$ so that $x_{i} k_{i}^{*}=x_{i} k_{i}$ for $i=1 \ldots s$. Let $h=\left(k_{1}, \ldots, k_{s} ; b\right) \in K \imath L$ for this choice of the $k_{i}$. Then $x h^{*}=$ $\left(x_{1} k_{1}^{*}, 1 b\right) \cup \cdots \cup\left(x_{s} k_{s}^{*}, s b\right)=\left(x_{1} k_{1}, 1 b\right) \cup \cdots \cup\left(x_{s} k_{s}, s b\right)=x h$ belongs to $R$. Hence $K$ is a relation group.

Proof of Corollaries 5.2 and 5.3 The first corollary is immediate. In Seress and Yang [15] the orbit closed groups of type $(K<L, \Omega)$ with both $(K, \Delta)$ and $(L, \Sigma)$ primitive are classified, when $(K, \Delta)$ has a regular set and $L$ does not contain $\operatorname{Alt}(\Sigma)$. Among these groups one may take for instance $(K, \Delta)=\left(C_{3}, 3\right)$ and any $L$ with $|\Sigma|>9$. As $\left(C_{3}, 3\right)$ is not a relation group Theorem 5.1 implies that $(K, L, \Omega)$ is not a relation group.

Next we are interested in conditions which guarantee that $K<L$ has a regular set provided $(K, \Delta)$ and $(L, \Sigma)$ have suitable regular sets.

Proposition 5.4 Let $\Delta$ be a set of size $d$ and let $\Sigma$ be a set of size $s$. Assume that $(K, \Delta)$ and $(L, \Sigma)$ are transitive permutation groups with regular sets of size $r_{\Delta} \neq \frac{d}{2}$ and $r_{\Sigma}$, respectively. Then $K$ ? $L$ has a regular set of size $r_{\Omega}=r_{\Sigma} d+\left(s-2 r_{\Sigma}\right)(d-$ $r_{\Delta}$ ) as an imprimitive group on $\Omega=\Delta \times \Sigma$.

Proof Let $x \subset \Delta$ be a regular set for $(K, \Delta)$ of size $r_{\Delta} \neq \frac{d}{2}$ and let $\{1, \ldots, k\} \subset \Sigma$ be a regular set for $(L, \Sigma)$ of size with $k=r_{\Sigma}$. Define $x^{\prime}=\Delta \backslash x$ and consider the following subset $w$ of $\Omega=(\Delta \times\{1\}) \cup \cdots \cup(\Delta \times\{s\})$ defined by

$$
w=(x \times\{1\}) \cup \cdots \cup(x \times\{k\}) \cup\left(x^{\prime} \times\{k+1\}\right) \cup \cdots \cup\left(x^{\prime} \times\{s\}\right) .
$$

Then we have $|w|=r_{\Sigma} d+\left(s-2 r_{\Sigma}\right)\left(d-r_{\Delta}\right)$.

We claim that $w$ is a regular sets for $K$ ? $L$. Let $h \in K$ ? $L$ with $w^{h}=w$. Since $h$ preserves the blocks and since $|x| \neq\left|x^{\prime}\right|$ it follows that $h$ permutes the block set $\{(x \times\{1\}) \cup \cdots \cup(x \times\{k\})\}$ and $\left\{\left(x^{\prime} \times\{k+1\}\right) \cup \cdots \cup\left(x^{\prime} \times\{s\}\right)\right\}$ separately. Since $\{1, \ldots, k\}$ and $\{k+1, \ldots, s\}$ are regular sets for $L$ we have $(x \times\{i\}) h=(x \times\{i\})$ for $1 \leq i \leq k$ and $\left(x^{\prime} \times\{j\}\right) h=\left(x^{\prime} \times\{j\}\right)$ for $k+1 \leq j \leq s$. But as $(x \times\{i\})$ and $\left(x^{\prime} \times\{j\}\right)$ are regular sets for the $H$ action on $(\Delta \times\{i\})$ and $(\Delta \times\{j\})$, respectively, we find that $h$ is the identity on $\Delta \times\{1 . . s\}$.

By a similar argument we can show that $K$ \& $L$ has a regular set provided $K$ has sufficiently many sets in comparison to the degree of $L$.

Proposition 5.5 Let $(K, \Delta)$ and $(L, \Sigma)$ be permutation groups where $L$ is transitive. Assume that $K$ has at least $|\Sigma|$ regular sets on $\Delta$ of pairwise distinct size. Then $K \geq L$ has a regular set as an imprimitive group on $\Omega=\Delta \times \Sigma$. 
Proof Let $s:=|\Sigma|$ and let $x_{1}, \ldots, x_{s} \subset \Delta$ be regular sets for $K$ of pairwise distinct sizes. Then

$$
w=\left(x_{1} \times\{1\}\right) \cup \cdots \cup\left(x_{s} \times\{s\}\right)
$$

is a regular set for $K \geq L$ by the same reasoning as in the proof of Proposition 5.4.

Comment Trivially, if a group of degree $n$ admits regular sets then it has regular sets of size $\leq \frac{n}{2}$, just replace a set by its complement. In particular, in Proposition 5.4 one may select regular sets to have size $r_{\Delta}<\frac{d}{2}$ and $r_{\Omega}<\frac{d s}{2}$ provided $r_{\Sigma} \neq \frac{s}{2}$.

\section{Imprimitive groups}

Let $H$ be an imprimitive permutation group on $\Omega$ and let $\Omega_{1}, \ldots, \Omega_{s}$ with $\Omega=$ $\Omega_{1} \cup \cdots \cup \Omega_{s}$ be a system of blocks of imprimitivity. (As usual, an imprimitive group is transitive.) Then $H$ induces permutation groups $K:=H_{\Delta}^{\Delta}$ on $\Delta:=\Omega_{1}$ and $L:=H^{\Sigma}$ on $\left\{\Omega_{1}, \ldots, \Omega_{s}\right\}$ where $\Sigma:=\{1, \ldots, s\}$. In particular, $H \subseteq K<L$ acts in the imprimitive action on $\Omega=\Delta \times \Sigma$. Evidently $K$ is primitive on $\Delta$ if and only if $\Delta$ is a minimal (non-trivial) block of imprimitivity of $H$. If $R$ is a relation recall that $\operatorname{ar}(R)=\{|x|: x \in R\}$ is the arity of $R$ and the maximum set size in $R$ is $m(R):=\max \{|x|: x \in R\}$.

Theorem 6.1 Let $H$ be an imprimitive permutation group on $\Omega$ and let $\Delta \subset \Omega$ be a minimal block of imprimitivity. Suppose that $H_{\Delta}^{\Delta}$ is a relation group on $\Delta$ which has at least $\frac{|\Omega|}{|\Delta|}$ regular sets of pairwise distinct size. Then every subgroup of $H$ is a relation group.

Proof We put $K:=H_{\Delta}^{\Delta}, d:=|\Delta|$ and $n:=|\Omega|$. Let $R^{\Delta}$ be a relation on $\Delta$ with $K=\mathcal{G}\left(R^{\Delta}\right)$. Let $\Sigma$ be a set of size $s:=\frac{n}{d}$, hence $\Omega=\Delta \times \Sigma$, and let $R^{\Delta_{i}}$ be a copy of $R^{\Delta}$ on $\Delta \times\{i\}$. Then $H \subseteq \bar{H}:=K 2 \operatorname{Sym}(\Sigma)$ and $\bar{H}=\mathcal{G}\left(R^{\prime}\right)$ where $R^{\prime}$ is the relation defined in (1) in the proof of Theorem 5.1. All sets in $R^{\prime}$ have size $\geq(s-1) d+2>\frac{n}{2}$ since $s \geq 2$. It follows from Proposition 5.5 that $\bar{H}$ has a regular set of size $\leq \frac{n}{2}$. Now apply the first part of the Basic Lemma.

Asymptotically most sets in a primitive permutation not containing the alternating group of the same degree are regular. More precisely we have the following:

Corollary 6.2 There is a function $d(s)$ so that the following is true. Let $H$ be an imprimitive permutation group of degree $n$ with a block $\Delta$ of imprimitivity so that

(i) $H_{\Delta}^{\Delta}$ does not contain $\operatorname{Alt}(\Delta)$,

(ii) $|\Delta| \geq d(s)$ for $s=\frac{n}{|\Delta|}$.

Then every subgroup of $H$ is a relation group.

Proof We sketch the argument. Let $s$ be fixed. Then there is a bound $d(s)$ so that any primitive group $(K, \Delta)$ not containing $\operatorname{Alt}(\Delta)$ with $|\Delta| \geq d(s)$ has at least $s$ regular 
sets on $\Delta$ of pairwise different size. This is shown in Theorem 7.5 in Sect. 7 for $s=2$ and this argument extends readily to arbitrary $s$. Now apply Theorem 6.1.

Theorem 6.3 Let $H$ be an imprimitive permutation group on $\Omega$ with a minimal block $\Delta \subset \Omega$ of imprimitivity. Put $K:=H_{\Delta}^{\Delta}, d:=|\Delta|$ and $n:=|\Omega|$. Let $(L, \Sigma)$ with $s:=|\Sigma|=\frac{n}{d}$ be the group induced by $H$ on the blocks of imprimitivity. Suppose that

(i) $K$ is a primitive relation group and has a regular set of size $r_{\Delta} \neq \frac{d}{2}$ on $\Delta$, and

(ii) $L$ is a relation group and L has a regular set on $\Sigma$.

Then every subgroup of $H$ is a relation group on $\Omega$.

Proof We follow the proof of Theorem 5.1 using the same terms. Let $R$ and $R^{\prime \prime}$ be as in (2) and (3), and let $w$ be the regular set for $K$ ? $L$ as in (4). Let $G \subseteq K$ ? $L$. We put $R^{G}=w^{G}$, the orbit of $w$ under $G$, and consider

$$
G^{\prime}:=\mathcal{G}\left(R \cup R^{\prime \prime} \cup R^{G}\right) .
$$

Clearly $G \subseteq G^{\prime}$ and it remains to show the converse. First note that a set in $R$ has size $\leq d-2$ while sets in $R^{\prime \prime}$ have size $\ell d$ with $1 \leq \ell \leq m_{\Sigma}$. The set $w$ has size $>d$. Therefore $R$ is preserved by $G^{\prime}$ as a set and so

$$
G^{\prime}=\mathcal{G}(R) \cap \mathcal{G}\left(R^{\prime \prime} \cup R^{G}\right) \subseteq K \imath \operatorname{Sym}(\Sigma),
$$

by the proof above of Theorem 5.1. Let $h$ be an element of $G^{\prime}$. If $h$ leaves $R^{G}$ invariant then also $R^{\prime \prime}$ is invariant under $h$ so that $h \in \mathcal{G}\left(R \cup R^{\prime \prime}\right)=K$ ? $L$. This means that there is some $g \in G$ with $w^{h}=w^{g}$ so that $h g^{-1} \in K$ 乙 $L$ stabilizes $w$. Since $w$ is a regular set for $K$ ? $L$ we have $h=g \in G$.

Hence we have to consider the situation where $h$ does not leave $R^{G}$ invariant. We claim that this is impossible. Let $u \in R^{G}$ with $u^{h} \in R^{\prime \prime}$. Since $G \subseteq K$ ? $L$ it follows that $u$ decomposes over the $\Delta_{i}$ in the same way (4) as $w$ so that

$$
u=u_{1} \cup \cdots \cup u_{k} \cup u_{k+1}^{\prime} \cup \cdots \cup u_{s}^{\prime},
$$

where $2 \leq\left|u_{1}\right|=\cdots=\left|u_{k}\right|=r_{\Delta} \leq d-2$ and $d-r_{\Delta}=\left|u_{k+1}\right|=\cdots=\left|u_{s}\right|$. Since $h \in K$ i $\operatorname{Sym}(\Sigma)$ preserves the block structure also $u^{h}$ decomposes in this way. But as $u^{h} \in R^{\prime \prime}$ this is in contradiction to (3).

\section{Some classes of imprimitive relation groups}

Let $H$ be an imprimitive permutation group on $\Omega$ and let $\Delta^{(0)}$ be some minimal (nontrivial) block of imprimitivity of $H$. Let $K^{(0)}$ be the group induced by $H$ on $\Delta^{(0)}$ and let $L$ be the group induced by $H$ on the $H$-images of $\Delta^{(0)}$. Then $H \subseteq K^{(0)}$ ₹ $L$.

By repeating the same process for $L$ we obtain a chain $\left(K^{(0)}, \Delta^{(0)}\right), \ldots,\left(K^{(t)}, \Delta^{(t)}\right)$ of primitive groups so that $H$ is contained in the iterated wreath product,

$$
H \subseteq K^{(0)} \prec\left(K^{(1)} \prec\left(\ldots 2\left(K^{(t-1)} \prec K^{(t)}\right) \ldots\right)\right) .
$$


We call $\left(K^{(0)}, \Delta^{(0)}\right), \ldots,\left(K^{(t)}, \Delta^{(t)}\right)$ with $t \geq 1$ a imprimitivity chain of length $t$ for $H$. As there is no Jordan-Hölder Theorem for primitivity a group may have several incomparable imprimitivity chains, including chains of different lengths.

We say that $H$ is $\mathcal{A}$-imprimitive if there is a collection $\mathcal{A}$ of primitive permutation groups and some imprimitivity chain $\left(K^{(0)}, \Delta^{(0)}\right), \ldots,\left(K^{(t)}, \Delta^{(t)}\right)$ for $H$ so that $\left(K^{(i)}, \Delta^{(i)}\right)$ belongs to $\mathcal{A}$ for all $i=0 \ldots t$. Similarly, $H$ is $\mathcal{A}^{\prime}$-imprimitive if there is an imprimitivity chain $\left(K^{(0)}, \Delta^{(0)}\right), \ldots,\left(K^{(t)}, \Delta^{(t)}\right)$ for $H$ so that $\left(K^{(i)}, \Delta^{(i)}\right)$ does not belong to $\mathcal{A}$ for all $i=0 \ldots t$. (In both cases the containment is up to permutation isomorphisms.) Note that a group may happen to be $\mathcal{A}$-imprimitive and $\mathcal{A}^{\prime}$ imprimitive at the same time.

In view of Theorem 6.3 we are interested in those primitive groups which have regular sets, all of the same cardinality,

$$
\mathcal{L}_{\mathrm{SR}}:=\left\{(K, \Delta) \text { is primitive with regular sets, all of size } \frac{1}{2}|\Delta|\right\} .
$$

Now let

$$
\begin{aligned}
\mathcal{A}:= & \{\operatorname{Sym}(\Delta):|\Delta| \geq 2\} \cup\{\operatorname{Alt}(\Delta):|\Delta| \geq 3\} \\
& \cup \mathcal{L}_{\mathrm{NR}} \cup \mathcal{L}_{\mathrm{SR}} \\
& \cup\left\{\left(5, C_{5}\right),\left(8,2^{3}: 7\right),\left(9,3^{2}: 8\right)\right\} .
\end{aligned}
$$

It is easy to check that the list $\mathcal{L}_{\mathrm{ST}}$ and $\mathcal{L}_{\mathrm{OE}}$ from Sect. 4 are contained in $\mathcal{A}$. Now we have the following

Theorem 7.1 Let $H$ be an $\mathcal{A}^{\prime}$-imprimitive group on $\Omega$. Then all subgroups of $H$ are relation groups on $\Omega$.

Proof Let $H \subseteq K^{(0)}$ 々 $\left(K^{(1)} 2\left(\ldots 2\left(K^{(t-1)}>K^{(t)}\right) \ldots\right)\right)$ be an imprimitivity chain for $H$ where $\left(K^{(i), \Delta^{(i)}}\right)$ is not in $\mathcal{A}$, for all $i=0 \ldots t$. As the list $\mathcal{L}$ from Sect. 4 is contained in $\mathcal{A}$ it follows from Theorem 4.2 that $\left(K^{(i)}, \Delta^{(i)}\right)$ is a relation groups for all $i=0 \ldots t$. In addition, all $\left(K^{(i)}, \Delta^{(i)}\right)$ have regular sets of cardinality $\neq \frac{1}{2}\left|\Delta^{(i)}\right|$. It follows by induction from Proposition 5.4 that $\left(K^{(1)} 2\left(\ldots 2\left(K^{(t-1)} 2 K^{(t)}\right) \ldots\right)\right)$ is a relation group with a regular set, see the comment following Proposition 5.5. Now use Theorem 6.3.

Before we turn to the groups in $\mathcal{L}_{\mathrm{SR}}$ specifically we are able to state several corollaries which do not require the explicit knowledge of this class of groups.

Odd degree and odd order A primitive group of odd degree with at least one regular set automatically has two regular sets of different sizes. Therefore

Corollary 7.2 Let $H$ be an $\mathcal{O}^{\prime}$-imprimitive group of odd degree where $\mathcal{O}=$ $\{\operatorname{Sym}(\Delta), \operatorname{Alt}(\Delta):|\Delta| \geq 3\} \cup \mathcal{L}_{\mathrm{NR}} \cup\left\{\left(5, C_{5}\right),\left(9,3^{2}: 8\right)\right\}$. Then all subgroups of $H$ are relation groups. 
In particular,

Corollary 7.3 Let $H$ be an $\mathcal{O}^{\prime}$-imprimitive group of odd order where $\mathcal{O}=\left\{\left(3, C_{3}\right)\right.$, $\left.\left(5, C_{5}\right)\right\}$. Then all subgroups of $H$ are relation groups.

Solvable groups The regular sets of primitive solvable groups have been studied in Gluck [8]. From his result it follows that the solvable groups belonging to $\mathcal{A}$ form the list

$$
\begin{aligned}
\mathcal{S}:= & \{\operatorname{Sym}(\Delta), \operatorname{Alt}(\Delta):|\Delta| \leq 4\} \\
& \cup\left\{\left(5, D_{10}\right),\left(7, F_{42}\right),\left(8,2^{3}: 7.3\right),\left(9,3^{2}:\left(2 . L_{2}(3)\right),\right.\right. \\
& \left.\left(9,3^{2}: 2 . L_{2}(3) \cdot 2\right),\left(9,3^{2}: D_{8}\right),\left(9,3^{2}: 8.2\right)\right\} \\
& \cup\left\{\left(5, C_{5}\right),\left(8,2^{3}: 7\right),\left(9,3^{2}: 8\right)\right\} .
\end{aligned}
$$

Therefore the theorem provides us with the following.

Corollary 7.4 Let $H$ be a solvable $\mathcal{S}$-imprimitive group. Then all subgroups of $H$ are relation groups.

Using much the same idea as in [5] we show that $\mathcal{L}_{\mathrm{SR}}$ is finite, and this will conclude the discussion of this particular class of groups. In a forthcoming paper we will determine $\mathcal{L}_{\text {SR }}$ explicitly.

Theorem 7.5 Let $G$ be a primitive permutation group on $\Omega$ and assume that $G$ does not contain $\operatorname{Alt}(\Omega)$. Then with only finitely many exceptions $G$ has regular sets of cardinality $k_{1} \neq k_{2}$.

Proof This follows from Theorem 2.2 if $n=|\Omega|$ is odd. So let $n=2 k$ and denote the minimal degree of $G$ by $m$, so that $m$ is the least number of elements in $\Omega$ moved by a non-identity element of $G$. Hence a non-identity element fixes at most $2^{n-\frac{m}{2}}$ subsets of $\Omega$. Therefore the cardinality of the set

$$
A=\left\{(\Delta, g):|\Delta| \neq k, \Delta^{g}=\Delta, g \neq 1\right\}
$$

can be bounded by

$$
2^{n}-\left(\begin{array}{l}
n \\
k
\end{array}\right) \leq|A| \leq 2^{n-\frac{m}{2}}(|G|-1) .
$$

As the number of odd-sized (or even-sized) subsets of an $n$-element set is $2^{n-1}$ we have

$$
2^{n-1} \leq 2^{n}-\left(\begin{array}{l}
n \\
k
\end{array}\right) \leq 2^{n-\frac{m}{2}}|G|
$$


In particular, $2^{\frac{m}{2}-1} \leq|G|$, and from the well-known bound $2^{\frac{n}{m}} \leq|G|$, see for instance the Proposition in [5], we have

$$
\frac{n}{2} \leq \log |G| \cdot(\log |G|+1) .
$$

In particular

$$
n^{1+\left(\log _{2} n\right)}<|G|
$$

for all sufficiently large $n$. Using Maróti's estimate in [13] for the order of a primitive groups it follows that $G$ is a subgroup of $\operatorname{Sym}_{s} 2 \operatorname{Sym}_{t}$ acting in the product action of degree $\left(\begin{array}{l}s \\ a\end{array}\right)^{t}$ where $\operatorname{Sym}_{s}$ acts naturally on the $a$-element subsets of $\{1 \ldots s\}$, for some $a$ and $t \geq 2$. In Lemma 3.1 of [15] it is shown that in this action $\operatorname{Sym}_{s} 2 \operatorname{Sym}_{t}$ has regular sets of at least four distinct sizes.

\section{Closing remarks and open problems}

We conclude with a few remarks and suggestions for further work on the two topics of this paper.

Relation groups From the results in Sects. 5-7 one should take the view that a transitive group in general tends to be a relation group, and that it may not be so easy to classify the imprimitive groups that are not relation groups. The only firm result to show that certain kinds of imprimitive groups are not relation groups is Theorem 5.1. A measure for a group $(G, \Omega)$ to be a relation group is

$$
r(G):=\min \{|\mathcal{G}(R): G| \text { where } R \text { is a relation on } \Omega \text { with } G \subseteq \mathcal{G}(R)\} .
$$

Thus $r(G)=1$ if and only if $G$ is a relation group.

- Is $r(G)$ bounded in terms of the length of an imprimitivity chain for $G$ ? Are there absolute bounds for good classes of imprimitive group?

- In all examples with $r(G)>1$ we are aware of it appears that $r(G)$ is even. Is this true for all imprimitive groups?

Orbit closure In the literature orbit closure properties have been considered almost exclusively for groups with imprimitivity chains of length $\leq 2$. (Groups with length 2 are the two-step imprimitive groups in [15].) The results here on relation groups imply closure properties for arbitrary subgroups in the presence of suitable regular sets. A measure for a groups $(G, \Omega)$ to be orbit closed is

$$
c(G):=\left|G^{*}: G\right|,
$$

where $G^{*}$ is the orbit closure of $G$. By Proposition 2.5 we have $G \subseteq G^{*} \subseteq \mathcal{G}(R)$ for any relation $R$ with $G \subseteq \mathcal{G}(R)$ and therefore $c(G)$ divides $r(G)$.

- The set-transitive group $G=L_{2}(8)$ of degree 9 in Lemma 2.1 has $c(G)=6$ !. This is the largest value for $c(G)$ that we are aware of. Is there an absolute bound for $c(G)$ ? 
- Considering Corollary 5.3, what are the groups $G$ with $1=c(G)<r(G)$ ?

Finally note that if $G \subseteq \mathcal{G}(R)$ for a relation $R$ then $G \subseteq G^{*} \subseteq \mathcal{G}(R)$ and hence $r(G)=c(G) \cdot r\left(G^{*}\right)$. This suggests that questions about relation groups can be reduced to orbit closed groups.

\section{References}

1. Albertson, M., Collins, K.: Symmetry breaking in graphs. Electron. J. Comb. 3, \#R18 (1996)

2. Bates, C., Bundy, D., Hart, S., Rowley, P.: Primitive k-free permutation groups. Arch. Math. 88, 193198 (2007)

3. Beaumont, R., Peterson, R.: Set-transitive permutation groups. Can. J. Math. 7, 35-42 (1955)

4. Betten, D.: Geometrische Permutationsgruppen. Mitt. Math. Ges. Hamb. 10, 317-324 (1977)

5. Cameron, P.J., Neumann, P.M., Saxl, J.: On groups with no regular orbits on the set of subsets. Arch. Math. 43, 295-296 (1984)

6. Dalla Volta, F.: Regular sets for the affine and projective groups over the field of two elements. J. Geom. 33, 17-26 (1988)

7. Dalla Volta, F.: Regular sets for projective orthogonal groups over finite fields of odd characteristic. Geom. Dedic. 32, 229-245 (1989)

8. Gluck, D.: Trivial set-stabilizers in finite permutation groups. Can. J. Math. 35, 59-67 (1983)

9. Key, J.D., Siemons, J.: Regular sets and geometric groups. Results Math. 11, 97-116 (1987)

10. Key, J.D., Siemons, J.: On the k-closure of finite linear groups. Boll. Unione Mat. Ital. 7(1-B), 31-55 (1987)

11. Kisielewicz, A.: Symmetry groups of boolean functions and constructions of permutation groups. J. Algebra 199, 379-403 (1998)

12. Laflamme, C., Van Thé, N., Sauer, N.: Distinguishing number of countable homogeneous relational structures. Electron. J. Comb. 17, \#R20 (2010)

13. Maróti, A.: On the orders of primitive permutation groups. J. Algebra 258, 631-640 (2002)

14. Seress, A.: Primitive groups with no regular orbits on the set of subsets. Bull. Lond. Math. Soc. 29, 697-704 (1997)

15. Seress, A., Yang, K.: On orbit equivalent, two-step imprimitive permutation groups. Contemp. Math. 470, 271-285 (2008)

16. Siemons, J.: On partitions and permutation groups on unordered sets. Arch. Math. 38, 391-403 (1982)

17. Wielandt, H.: Permutation Groups Through Invariant Relations and Invariant Function, Ohio Lecture Notes. Mathematische Werke, vol. 1. Group Theory. de Gruyter, Berlin (1994) 